\title{
Cosmic infrared background and Population III: an overview
}

\author{
By A. Kashlinsky \\ Code 665, Observational Cosmology Lab, Goddard Space Flight Center, Greenbelt MD 20771 \\ e-mail: kashlinsky@milkyway.gsfc.nasa.gov
}

\begin{abstract}
We review the recent measurements on the cosmic infrared background (CIB) and their implications for the physics of the first stars era, including Population III. The recently obtained CIB results range from the direct measurements of CIB fluctuations from distant sources using deep Spitzer data to strong upper limits on the near-IR CIB from blazar spectra. This allows to compare the Population III models with the CIB data to gain direct insight into the era of the first stars and the formation and evolution of Population III and the microphysics of the feedback processes in the first halos of collapsing material. We also discuss the cosmological confusion resulting from these CIB sources and the prospects for resolving them individually with NASA's upcoming space instruments such as the JWST.
\end{abstract}

\section{Introduction}

The very first stars to form in the Universe, commonly called Population III, are now thought to have been very massive stars forming out of primordial metal-free gas at redshifts exceeding $z \sim 10$ (see recent review by Bromm \& Larson 2004). Assuming that the density field responsible for structure formation is given by the $\Lambda \mathrm{CDM}$ model, the first collapsing haloes hosting such stars may be too faint to be observed with the present telescopes. Their studies may, however, be possible via the cumulative radiation emitted by the first luminous objects, most of which by now has been shifted into the near-IR wavelengths of $\sim 1-10 \mu \mathrm{m}$. We review here the connection between the massive Population III stars and the cosmic infrared background measurements and the implications of the latter for the duration, nature and abundance of luminous objects that comprised the era of the first stars.

The cosmic infrared background (CIB) is a repository of emissions throughout the entire history of the Universe. Cosmic expansion shifts photons emitted in the visible/UV bands at high $z$ into the near-IR (NIR) and the high- $z$ NIR photons appear today in midto far-IR. Consequently, the NIR part of the CIB spectrum $(1 \mu \mathrm{m}<\lambda<10 \mu \mathrm{m})$ probes the history of direct stellar emissions from the early Universe, and the longer wavelengths contain information about the early dust production and evolution. The recent years have seen significant progress in CIB studies, both in identifying and/or constraining its mean level (isotropic component) and fluctuations (see Kashlinsky 2005a for a recent review).

The CIB contains emissions also from objects inaccessible to current (or even future) telescopic studies and can, therefore, provide unique information on the history of the Universe at very early times. One particularly important example of such objects are Population III stars (hereafter Pop III), the still elusive zero-metallicity stars expected to have preceded normal stellar populations seen in the farthest galaxies to-date. Throughout this review we will use the term "era of the first stars", or "Pop III era", with the understanding that the actual era may be composed of objects of various nature from purely zero-metallicity stars, to low- metallicity stars to even possibly mini-quasars whose contribution to the CIB is driven by energy released by gravitational accretion, as opposed to stellar nucleosynthesis. 
Pop III stars are thought to have preceded the normal metal-enriched stellar populations, but even if massive and luminous they are inaccessible to direct observations by current telescopes because of the high redshits at which they are located. Extensive numerical investigations, as discussed by Bromm and Norman in this meeting, suggest that they had to be very massive, forming out of density fluctuations specified by the standard $\Lambda \mathrm{CDM}$ model. If massive, they are expected to have left a significant level of diffuse radiation shifted today into the IR, and it was suggested that the CIB contains a significant contribution from Pop III in the near-IR, manifest in both its mean level and its anisotropies (e.g. Santos et al 2002, Salvaterra \& Ferrara 2003, Cooray et al 2004, Kashlinsky et al 2004; see also review by Kashlinsky 2005a). This notion has recently received strong support from measurements of CIB anisotropies in deep Spitzer/IRAC images (Kashlinsky et al 2005) as will be discussed below.

The structure of this review is as follows: Sec. 2] summarizes the current measurements of the mean levels of the CIB and the contributions to them from the observed galaxy populations. Sec. 3 discusses the theoretical connection between the CIB and the first (massive) stars and Sec. 4 gives the limits on the near-IR CIB from measurements of absorption in the high-energy spectra of cosmological sources. Sec. 5 reviews the recent Spitzer-based measurements of CIB fluctuations from early epochs and their interpretation in terms of the nature and the epochs of the contributing sources. Finally, the prospects for resolving these sources in future measurements are summarized in Sec. 6] followed with conclusions in Sec. 7

The $\mathrm{AB}$ magnitude system is adopted throughout; the conversion to fluxes is simple as zero $\mathrm{AB}$ magnitude corresponds to the flux of $3631 \mathrm{Jy}$.

\section{Direct CIB measurements}

Galactic and Solar System foregrounds are the major obstacles to space-based CIB measurements. Galactic stars are the main contributors at near-IR $(<$ a few $\mu \mathrm{m})$, zodiacal light from the dust in the Solar system dominates between $\sim 10$ and $\sim 50 \mu \mathrm{m}$, and Galactic cirrus emission produces most of the foreground at IR wavelengths at $>50$ $\mu \mathrm{m}$. Accurately removing the foregrounds presents a challenge and many techniques have been developed to do this as well as possible. Stars can be removed in surveys with fine angular resolution or by statistically extrapolating the various stellar contributions to zero. Zodiacal light contributions usually are removed using the DIRBE zodi model or its derivatives. Galactic cirrus and zodiacal light are both intrinsically diffuse, but are fairly homogeneous adding to the effectiveness of CIB fluctuations studies at mid- to far-IR (Kashlinsky et al 1996a,b).

In the near-IR CIB detections are difficult because of the substantial foreground by Galactic stars. Claims of detections of the mean isotropic part of the CIB are based on various analyses of DIRBE and IRTS data (Dwek \& Arendt 1998, Matsumoto et al 2005, Wright \& Reese 2000, Gorjian et al 2001, Cambresy et al 2001). The measurements agree with each other, although the methods of analysis and foreground removal differ substantially. They also agree with the measured amplitude of CIB fluctuations using DIRBE data (Kashlinsky \& Odenwald 2000). The results seem to indicate fluxes significantly exceeding those from observed galaxy populations. Fig. [1]summarizes the current CIB measurements. Only the near-IR CIB at $\lambda<10 \mu \mathrm{m}$ is relevant for the discussion that follows as we will not discuss here the possible dust contribution from the high $z$ contributing to (re)emissions contributing to CIB at longer wavelengths.

One can compare the claimed CIB levels to the CIB levels obtained from integrating the contributions from galaxies seen in faint galaxy counts surveys. The net fluxes per 


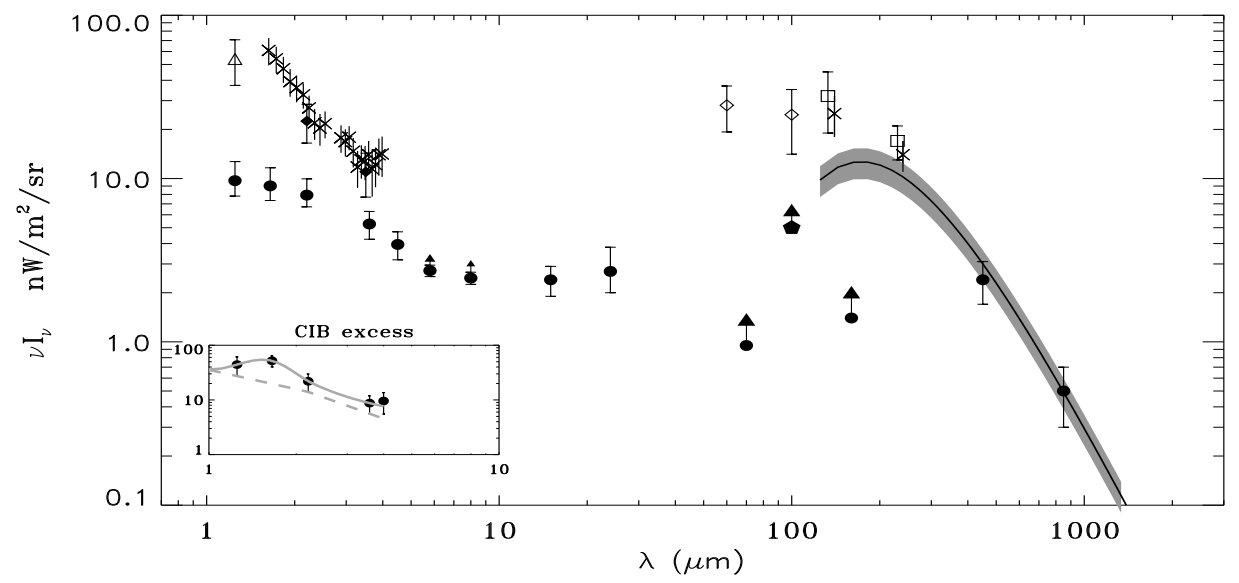

Figure 1. CIB and "ordinary" galaxy (OG) contributions (filled circles) vs wavelength. The numbers are adopted from Fig. 9 of Kashlinsky (2005a and references therein) and are discussed at length there. Briefly, the CIB fluxes from IRTS measurements are shown with crosses from Matsumoto et al (2005), diamonds are from Gorjian et al (2000) at $2.2 \mu \mathrm{m}$, and from Dwek \& Arendt (1998) and Wright \& Reese (2000) at $3.5 \mu \mathrm{m}$. The flux from ordinary galaxies is shown with filled circles and is taken from HST counts out to $2.2 \mu \mathrm{m}$ (Madau \& Pozzetti 2000) and from Spitzer/IRAC counts at 3.6 and $4.5 \mu \mathrm{m}$ (Fazio et al 2004). At $\lambda>10 \mu \mathrm{m}$ no CIB excess was observed and the levels of CIB are consistent with the net contribution from OG. The inset shows the NIRBE spectrum, $\nu I_{\nu}$ in $\mathrm{nW} / \mathrm{m}^{2} / \mathrm{sr}$ vs $\lambda$ in $\mu \mathrm{m}$, from Kashlinsky (2005a). The two thick light-shaded lines show the ranges of the NIRBE spectrum suggested by the data: the solid line goes through the central points, while the dashed line grazes the lower edges of the data.

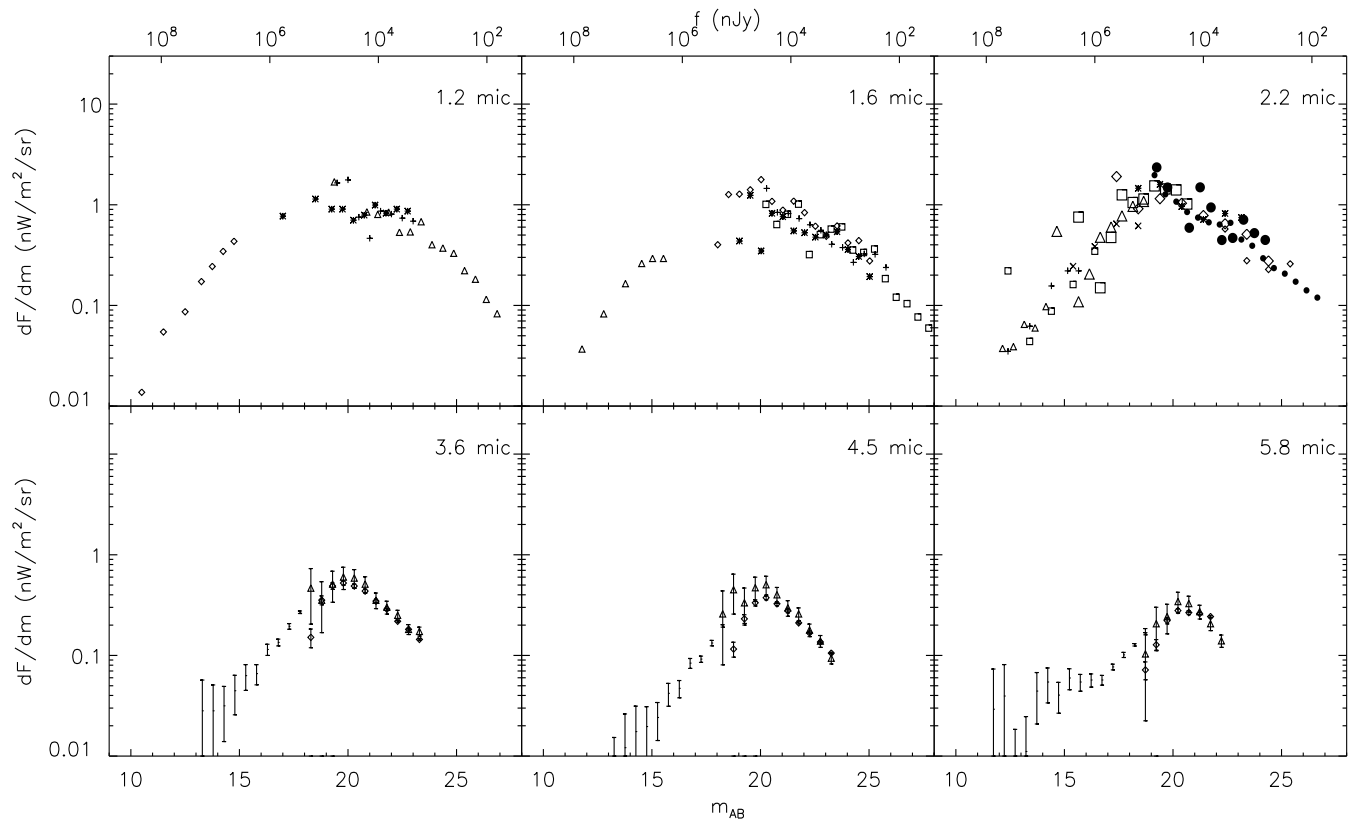

Figure 2. Cumulative flux in $\mathrm{nW} / \mathrm{m}^{2} / \mathrm{sr}$ contributed by galaxies from a narrow $d m$ magnitude bin for HST (Madau \& Pozzetti 2000 - upper panels) and Spitzer-based (Fazio et al 2004) counts data. The points are adopted from Kashlinsky (2005a - see Figs. 15,16). 
magnitude interval $d m$ from such counts are given by:

$$
\frac{d F}{d m}=f(m) \frac{d N}{d m}
$$

where $d N / d m$ is the differential counts of galaxies per unit solid angle in the magnitude bin $d m$ and $f(m)$ is the flux corresponding to $m$.

Fig. 2] shows the near-IR CIB flux contributions per $d m$ from the galaxy populations observed in various deep surveys. The total NIR fluxes by these "ordinary" galaxies (OG) from the HST and Spitzer IRAC measurements are shown as filled circles in Fig. 1 these total fluxes saturate at $\mathrm{AB}$ magnitude of about 20 and at IR wavelengths shorter than $\sim 5-8 \mu \mathrm{m}$ they appear to be lower by a significant factor than the detected CIB.

The difference between the claimed CIB levels and the total fluxes from "ordinary" (i.e. not Pop III) galaxies are known as the near-IR background excess (hereafter NIRBE), whose integrated amount between 1 and $4 \mu \mathrm{m}$ is (Kashlinsky 2005a):

$$
F_{\text {NIRBE }}(1-4 \mu \mathrm{m})=29 \pm 13 \mathrm{nW} / \mathrm{m}^{2} / \mathrm{sr}
$$

\section{Cosmic infrared background excess and Population III}

It was suggested that the NIR CIB excess is produced by massive Pop III stars at high $z(>8-10)$ (Santos et al 2002, Salvaterra \& Ferrara 2003, Magliochetti et al 2003 Cooray et al 2004 Kashlinsky et al 2004). Because Pop III stars, if massive, would radiate at the Eddington limit, where $L \propto M$, the levels of the total flux produced by them are largely model-independent (Rees 1978, Kashlinsky et al 2004) leading to a robust prediction for the total bolometric flux from them. For completeness we reproduce briefly the argument from Kashlinsky et al (2004): Each star would produce flux $\frac{L}{4 \pi d_{L}^{2}}$, where $d_{L}$ is the luminosity distance. Because for massive stars $L \propto M$, the total comoving luminosity density from Pop III is $\int n(L) L d L \propto \Omega_{\text {baryon }} f_{*} \frac{3 H_{0}^{2}}{8 \pi G}$, where $n(L)$ is their luminosity function and $f_{*}$ is the mass fraction of baryons locked in Pop III stars at any given time. In the flat Universe, the volume per unit solid angle subtended by cosmic time $d t$ is $d V=c(1+z) d_{L}^{2} d t$. Finally, these stars would radiate at efficiency $\epsilon(\simeq 0.007$ for hydrogen burning). This then leads to the closed expression for the total bolometric flux from these objects:

$$
F_{\text {bol }}=\frac{3}{8 \pi} \frac{c^{5} / G}{4 \pi R_{H}^{2}}\left\langle(1+z)^{-1}\right\rangle \epsilon f_{3} \Omega_{\text {baryon }} \simeq 4 \times 10^{7} z_{3} \epsilon f_{3} \Omega_{\text {baryon }} h^{2} \frac{\mathrm{nW}}{\mathrm{m}^{2} \mathrm{sr}}
$$

Here $f_{3}$ is the mean mass fraction of baryons locked in Pop III stars and $z_{3} \equiv \frac{1}{\left\langle(1+z)^{-1}\right\rangle}$ is a suitably averaged redshift over their era. The total flux is a product of the maximal luminosity produced by any gravitational process, $c^{5} / G$, distributed over the surface of the Hubble radius, $R_{H}=c H_{0}^{-1}$, and the fairly understood dimensionless parameters. The term $c^{5} / G$ appears because the emissions are produced by the nuclear burning of stars evolving in gravitational equilibrium with the (radiation) pressure (or gravitational accretion as in the case of mini-quasars). From WMAP observations we adopt $\Omega_{\text {baryon }} h^{2}=0.044$ (Spergel et al 2006) and, because the massive stars are fully convective, their efficiency is close to that of hydrogen burning (Schaerer 2002, Siess et al 2002), $\epsilon=0.007$.

Requiring that the massive Pop III stars are responsible for the flux given by eq. 2.2 leads to the fraction of baryons locked in them of:

$$
f_{3}=(4.2 \pm 1.9) \times 10^{-3} z_{3} \frac{0.044}{\Omega_{\text {baryon }} h^{2}} \frac{0.007}{\epsilon}
$$


Within the uncertainty of eq. 2.2 only $\sim 2 \%$ of the baryons had to go through Pop III in order to produce the entire NIRBE. This is not unreasonable considering that primordial clouds are not subject to many of the effects inhibiting star formation at the present epochs, such as magnetic fields, turbulent heating etc. The only criterion for Pop III formation seems to be that primordial clouds turning-around out of the "concordance" $\Lambda \mathrm{CDM}$ density field have the virial temperature, $T_{\text {vir }}$, that can enable efficient formation of and cooling by molecular hydrogen (Abell et al 2002, Bromm et al 1999). Assuming spherical collapse of gaussian fluctuations and the $\Lambda$ CDM model from WMAP observations (Spergel et al 2006) the fraction of collapsed haloes at $z=10$ with $T_{\text {vir }} \geqslant(400,2000) \mathrm{K}$ is $(2.6,5) \times 10^{-2}$, which is in good agreement with eq. 3.2 These stars would have to be dominated by masses $>240 M_{\odot}$ to be consistent with low metallicities observed in Population II (Heger et al 2003).

The fraction given by eq. 3.2 was evaluated by using the bolometric CIB excess integrating from 1 to $4 \mu \mathrm{m}$. If there were significant amounts of CIB excess flux missed outside that range, the fraction $f_{3}$ would increase. However, at wavelengths $<0.1 z_{3} \mu \mathrm{m}$ the emission from the early times would be below the Lyman break and would be reprocessed to $\lambda>1 \mu \mathrm{m}$ (Santos et al 2002). If significant Pop III activity continued at $z_{3}<10$, which is unlikely, the rest frame Lyman break may be redshifted to $<1 \mu \mathrm{m}$, but the possible extra CIB excess from $<1 \mu \mathrm{m}$ will be compensated for by $f_{3}$ in eq. 3.2 decreasing with $z_{3}$. At longer wavelengths, the CIB excess given by eq. 2.2 can at most increase $f_{3}$ by $\sim 30 \%$. Thus the entire NIRBE can be explained if $2-4 \%$ of the baryons have been converted into massive Pop III stars at $z>10$ (Madau \& Silk 2005, Kashlinsky 2005b). This fraction of converted baryons scales linearly with the amplitude of $F_{\text {NIRBE }}$.

\section{CIB excess and high energy gamma-ray absorption}

An independent limit on the net amount of the isotropic component of the CIB comes from the measurements of absorption of high-energy cosmological sources. The latter results from the two-photon absorption, $\gamma \gamma_{\mathrm{CIB}} \rightarrow e^{+} e^{-}$, effective at high gamma-ray energies, $E_{\gamma} E_{\gamma_{\mathrm{CIB}}}>\left(m_{e} c^{2}\right)^{2}$. Because the reaction is due to electromagnetic interaction, its cross section is of order $\sim$ (the electron radius, $\left.e^{2} / m_{e} c^{2}\right)^{2}$ (it peaks at $\sim \frac{1}{4} \sigma_{T}$ ) which leads to significant absorption over cosmological distances.

The recent results from two $z \sim 0.18$ blazar spectra measurements at $\mathrm{TeV}$ energies by the HESS collaboration (Aharonian et al 2006) and similar constraints using slightly lower $z$ blazars (Dwek et al 2005) indicate that the claimed NIRBE would lead to more attenuation at $\sim 1-2 \mathrm{TeV}$ than the known blazar physics would allow. The thick light-shaded lines in the left panel of Fig. 3 show the attenuation due to NIRBE and galaxy counts fluxes for the CIB going through the central points of the near-IR CIB measurements (solid thick light-shaded line) and through the lower edges of the data (dashed thick light-shaded line). Such high attenuation factors (in excess of $\sim 10^{3}$ ) at $\sim 1.5 \mathrm{TeV}$ have been argued against by the HESS results. This would indicate that barring any changes in blazar physics - the NIRBE levels may be smaller than given by eq. (2.2).

However, it is important to emphasize that the HESS data still require significant CIB fluxes from the Pop III era. Indeed, this is also shown in Fig. 3 where we computed the attenuation factors assuming that the NIRBE contribution from Pop III scales as $I_{\nu} \propto \nu^{2}$ with a Lyman limit cutoff corresponding to the Pop III era ending at $z_{3}=10$ and normalized to the shown levels of the NIRBE flux, $\Delta_{C I B}$ in $\mathrm{nW} / \mathrm{m}^{2} / \mathrm{sr}$. The attenuation due to CIB from ordinary galaxies alone (filled circles in Fig. 11) is shown as dotted line and is not enough to account for the attenuation in the HESS blazars. The attenuation 

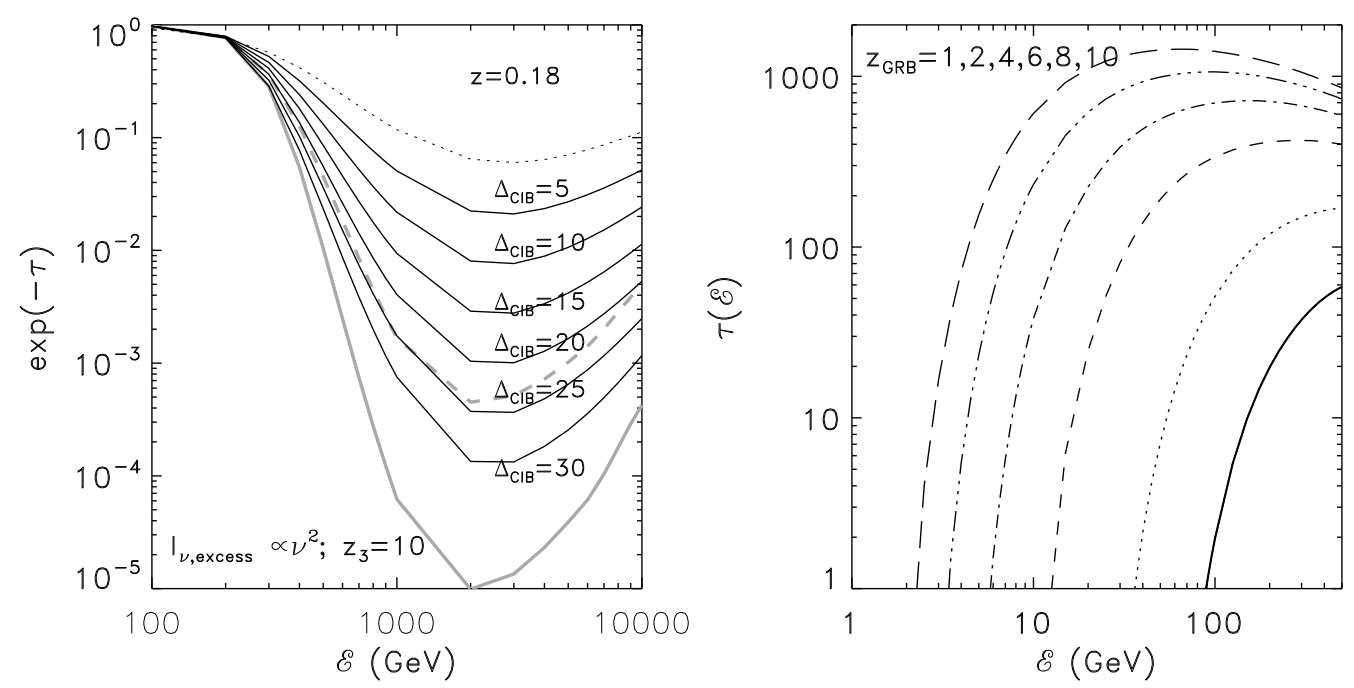

Figure 3. Left: Optical depth to photon-photon absorption for a source at $z=0.18$. The convention for the thick light-shaded lines corresponds to the inset in Fig. 1. Dotted line assumes only ordinary galaxies measured in deep counts and that their photons originated at $z \geqslant 0.18$. Solid lines correspond to the near-IR CIB excess from the Pop III era assuming it ended at $z_{3}=10$. The values of the excess in $\mathrm{nW} / \mathrm{m}^{2} / \mathrm{sr}$ are marked near the lines. Right: The net $\tau$ vs the GRB photon energy for the GRB redshifts shown in the panel. Solid, dotted, short-dashed, dash-dotted, dash-triple-dotted and long-dashed lines correspond to increasing order in $z$.

due to CIB levels claimed by the IRTS and DIRBE measurements is probably too strong. However, the figure shows that significant amounts of NIRBE are still allowed and required by the data. In particular the HESS data requires the levels of NIRBE due to Pop III (i.e. with Lyman cutoff in the CIB at $1 \mu \mathrm{m}$ ) to be below $\sim 15-20 \mathrm{nW} / \mathrm{m}^{2} / \mathrm{sr}$ still leaving up to a few percent of the baryons to have gone through Pop III.

This situation is expected to be resolved with the data from the upcoming GLAST mission which should measure spectra of high- $z$ gamma-ray bursts (GRBs) and blazars out to $300 \mathrm{GeV}$ (Kashlinsky 2005b). If Pop III at early epochs produced even a fraction of the claimed NIR CIB excess, they would provide a source of abundant photons at high $z$. The present-day value of $I_{\nu}=1 \mathrm{MJy} / \mathrm{sr}$ corresponds to the comoving number density of photons per logarithmic energy interval, $d \ln E$, of $\frac{4 \pi}{c} \frac{I_{\nu}}{h_{\text {Planck }}}=0.6 \mathrm{~cm}^{-3}$ and, if these photons come from high $z$, their number density would increase $\propto(1+z)^{3}$ at early times. These photons with the present-day energies, $E$, would also have had higher energies in the past: $E^{\prime}=E(1+z) \simeq(0.1-0.3)(1+z) \mathrm{eV}$. They would thus provide abundance of absorbers for sources of sufficiently energetic photons at high redshifts. Regardless of the precise amount of the NIR CIB from them, Pop III objects likely left enough photons to provide a large optical depth for high-energy photons from distant GRBs. The right panel in Fig. 3 comes from Kashlinsky (2005b) and shows the net optical depth (normalized to eq. 2.2) at high $z$. It shows that even if the NIRBE levels from Pop III were significantly smaller than in eq. 2.2 there should still be almost complete damping in the spectra of high- $z$ gamma ray sources at energies $<260(1+z)^{-2} \mathrm{GeV}$. Such damping should provide an unambiguous feature of the Pop III era and GLAST observations expected during the coming years would provide important information on the emissions from the Pop III era. 


\section{CIB fluctuations from deep Spitzer images and early populations}

Population III, if massive, should also have left significant CIB fluctuations providing a unique signature of their existence (Cooray et al 2004. Kashlinsky et al 2004). The reasons strong CIB fluctuations are as follows:

- If massive, each unit of mass in Pop III stars would emit several order of magnitude more luminosity than the present-day stellar populations.

- Pop III era is expected to have spanned a relatively short cosmic time $(<1$ Gyr $)$ leading to larger relative CIB fluctuations.

- Pop III stars are expected to have formed out of rare high- $\sigma$ peaks of the density field which would amplify their clustering.

We (Kashlinsky, Arendt, Mather, Moseley 2005) have attempted to measure these CIB fluctuations in deep Spitzer/IRAC data at 3.6 to $8 \mu \mathrm{m}$. The results of these measurements and their interpretation are discussed below. New and deeper measurements (Kashlinsky, Arendt, Mather, Moseley 2006, in preparation) support our earlier results.

\subsection{Observational results}

The main data used in Kashlinsky et al (2005; hereafter KAMM) came from the IRAC guaranteed-time-observations observations with $\sim 10$ hour integration of a field at high Galactic latitude. Additionally, the available data with shallower observations for 2 auxiliary fields were analyzed in order to test for isotropy of any cosmological signal. The datasets were assembled out of the individual frames using the least calibration method which has advantages over the standard pipeline calibration of the data in that the derived detector gain and offsets match the detector at the time of the observation, rather than at the time of the calibration observations.

For the final analysis we selected a subfield of $\simeq 5^{\prime} \times 10^{\prime}$ with a fairly homogeneous coverage. Individual sources have been clipped iteratively. The images were left with $>75 \%$ pixels for robust computation of the diffuse flux Fourier transforms. CIB fluctuations from Pop III at high $z$ should be independent of the clipping threshold, so the maps were also clipped progressively deeper to verify that our results are threshold-independent. As more pixels are removed, it becomes impossible to evaluate robust Fourier transforms, and then the diffuse flux correlation function was calculated. In that analysis we (Kashlinsky et al 2005) detected fluctuations significantly exceeding the instrument noise.

Fig. 4 shows the CIB fluctuation, after the power of the instrument noise has been subtracted, at $3.6 \mu \mathrm{m}$. The excess fluctuation on arcminute scales in the $3.6 \mu \mathrm{m}$ channel is $\sim 0.1 \mathrm{nW} / \mathrm{m}^{2} / \mathrm{sr}$; KAMM measure a similar amplitude in the longer IRAC bands indicating that the energy spectrum of the arcminute scale fluctuations is flat to slowly rising with increasing wavelength at least over the IRAC range of wavelengths. The detected signal is significantly higher than the instrument noise and the various systematics effects cannot account for it. There was a statistically significant correlation between the channels for the region of overlap suggesting the presence of the same cosmic signal in all channels. The correlation function at deeper clipping cuts, when too few pixels were left for Fourier analysis, remains roughly the same and is consistent with the power spectrum numbers.

Based on numerous tests KAMM show that the signal comes from the sky. Below are the possible sources of the fluctuations; they include origin in the spacecraft, the Solar system, the Galaxy and the (cosmological) sources outside the Galaxy:

1) Instrument noise was estimated in KAMM, who show that it is too low and has different pattern than the detected signal. Also, the cross-correlation between the channels for the overlap region of the data rules out significant instrumental contribution. 
A. Kashlinsky: Cosmic infrared background and early stars
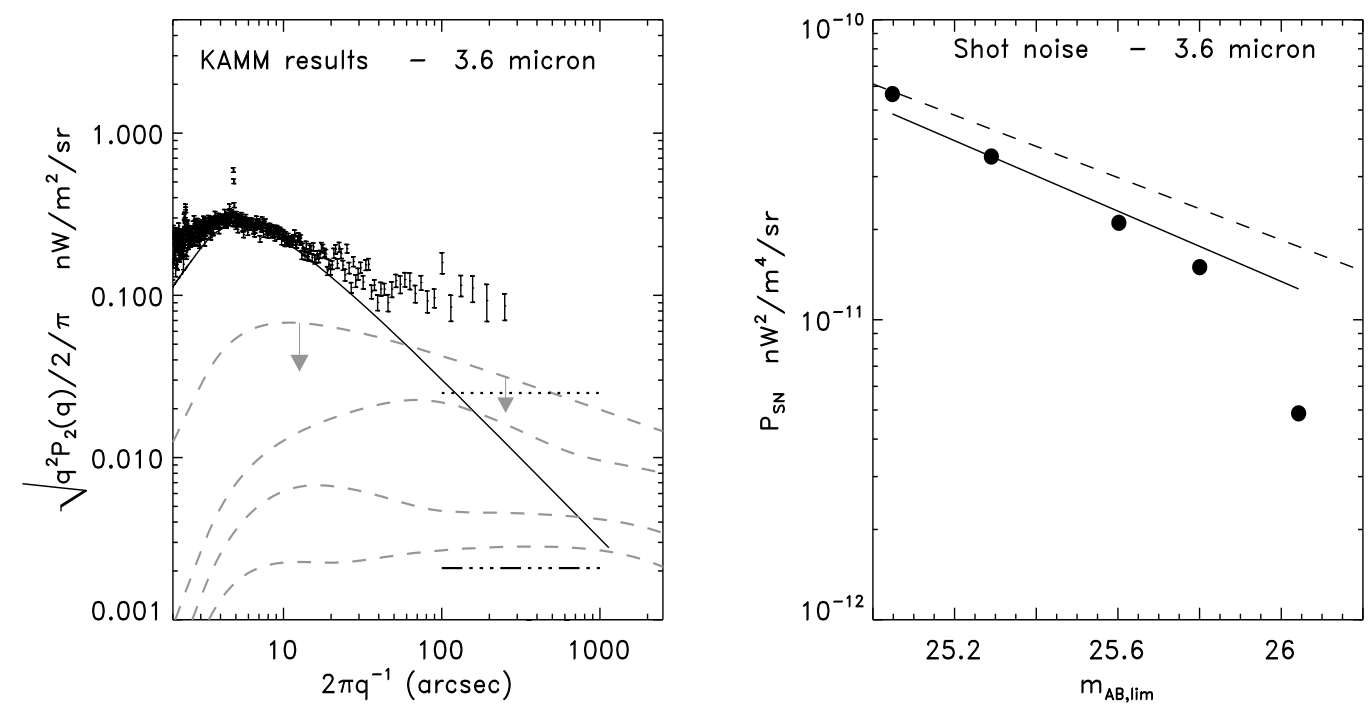

FigurE 4. Left: the CIB fluctuations spectrum measured by KAMM at $3.6 \mu \mathrm{m}$ are shown with 1- $\sigma$ error bars. Solid line shows the contribution from the shot noise from remaining ordinary galaxies. The upper limit on the zodiacal light fluctuations are shown with the dashed-triple-dotted line and on Galactic cirrus contribution with the dotted line. The upper limit on the contribution from ordinary galaxies is shown with the upper dashed line marked with down-pointing arrows. The lower dashed lines show more realistic contributions from ordinary galaxy populations as described in KAMM. Right: The amplitude of the shot noise power spectrum from remaining ordinary galaxies shown vs the limiting magnitude of galaxy removal by KAMM. Solid line is from the KAMM fit to the Spitzer counts (Fazio et al 2004); dashed line corresponds to counts analysis of Savage \& Oliver (2005)

2) Residual wings of removed sources also appear unlikely to have contributed significantly to the detected signal. KAMM have done extensive analysis and many tests to rule this out. E.g. the results remain the same for various clipping parameters, and for the various masks of the clipped-out sources as shown in their Supplementary Information.

3) Zodiacal light fluctuations were estimated by subtracting another data observed at two epochs separated by $\sim 6$ months. These are shown in Fig. 4 and are very small: the amplitude at $8 \mathrm{mic}$ is $<0.1 \mathrm{nW} / \mathrm{m}^{2} / \mathrm{sr}$ and assuming normal zodi spectrum the contribution to fluctuations would be totally negligible at shorter wavelengths.

4) Galactic cirrus is significant at channel $4(8 \mu \mathrm{m})$, which may in fact be dominated by the cirrus component (diffuse flux fluctuation $\sim 0.2 \mathrm{nW} / \mathrm{m}^{2} / \mathrm{sr}$ ), but given the energy spectrum of cirrus emission the other channels should have negligible cirrus. However, the map at Channel 4 correlates statistically significantly with the shorter wavelengths suggesting the presence of the same cosmic signal at $8 \mu \mathrm{m}$ as well.

5) Extragalactic sources thus seem the remaining logical explanation of the results. They include:

5.1) Ordinary galaxies with "normal" stellar populations

5.2) Population III with $M / L<<(M / L)_{\odot}$ and located at high $z$.

We discuss the constraints the data impose on the two cosmological components. More discussion is given with new measurements in Kashlinsky, Arendt, Mather \& Moseley (2006, in preparation). 


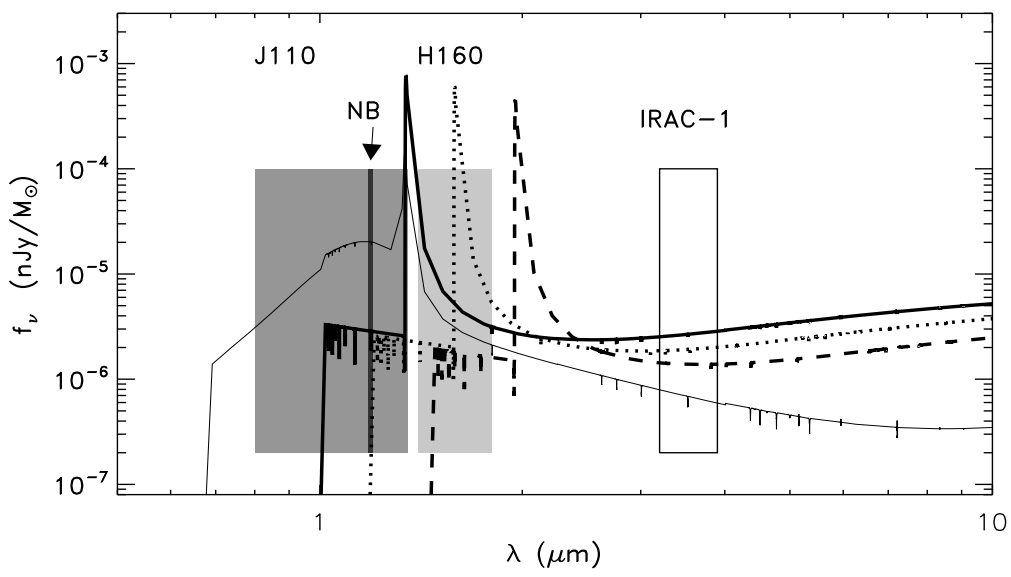

Figure 5. SED from Pop III system is shown for $z=10$ (thick solid line), 12 (dotted) and 15 (dashed). The lines are drawn from Santos et al (2002) for the case when processing of the radiation takes place in the gas inside the nebula. Thin solid line shows the SED from Santos et al for $z=10$ when the nebula is assumed transparent and the processing takes place inside the IGM. The J, NB, H and IRAC-3.6 $\mu$ m filters are shown. When the emission is reprocessed entirely by the IGM, the emission could extend below the Lyman limit at the rest-frame of the emitters, in which case many sources could have existed even at $z=10$, but escaped detection via the $J-N B>0.3$ criterion of ZEN (Willis \& Courbin 2005).

\subsection{Interpretation}

Extragalactic contributions to the CIB fluctuations come in two flavours: 1) the shot noise, and 2) due to clustering out of the primordial density fields which these sources trace. The shot-noise contribution, when evaluated directly from galaxy counts, gives a good fit to the fluctuations on smaller scales as shown in Fig. [ Ordinary galaxies have been eliminated from the maps down to very faint flux levels $(\sim 0.3 \mu \mathrm{Jy}$ in Channel 1). The remaining ordinary galaxies' contribution to the CIB mean levels is small $(<$ $0.1-0.2 \mathrm{nW} / \mathrm{m}^{2} / \mathrm{sr}$ ) and its fluctuation levels are shown in Fig. 团 On the other hand, the amplitude, power spectrum and the spectral energy dependence of the $>$ arcminutescale fluctuation can be explained by emissions from Pop III.

Fig. [5]shows the predicted flux due to spectral energy distribution (SED) of a Population III stellar system at $z=12-15$ and the region probed by the IRAC data. Although the Spitzer instruments cannot probe the peak of the emissions (due to Lyman emission), at the IRAC wavelengths we are still probing a region with substantial emissions by the Population III sources.

Any interpretation of the KAMM results must reproduce three major aspects:

- The sources in the KAMM data were removed to a certain (faint) flux limit, so the CIB fluctuations arise in populations with magnitudes fainter than the corresponding magnitude limit, $m_{\text {lim }}$.

- These sources must reproduce the excess CIB fluctuations by KAMM on scales $>0.5^{\prime}$.

- Lastly, the populations fainter than the above magnitude limit must account not only for the correlated part of the CIB, but - equally importantly - they must reproduce the (low) shot-noise component of the KAMM signal, which dominates the power at $<0.5^{\prime}$. 
Below we briefly discussion the constraints on the populations contributing to the KAMM signal in the above order:

1) Magnitude limits. The nominal limit above which sources have been removed in the KAMM analysis is $m_{\mathrm{AB}}=25.3$ at $3.6 \mathrm{um}$ and this by itself implies that the detected CIB fluctuations arise from fainter systems. At this magnitude one is already at the confusion limit for the IRAC beam at $3.6 \mu \mathrm{m}$ so fainter galaxies can be excised only by removing significantly more area in the map. The new mask then prevents computing reliably the power spectrum of the diffuse flux, which is why for deeper source removal KAMM presented its Fourier transform, the correlation function $C(\theta)$, in the Supplementary Information (SI). What is important in this context is that the $C(\theta)$, shown in Fig. SI-4 of the KAMM SI, does not change in shape and amplitude with additional clipping. At $3.6 \mu \mathrm{m}$ the extra clipping goes to the limit of $\simeq 140 \mathrm{nJy}$ for the faintest removed sources when KAMM stopped as only $6 \%$ of the map pixels remained. Thus the KAMM signal comes from sources fainter than $m_{\mathrm{AB}}>26.1$. For reference, this magnitude limit corresponds to $6 \times 10^{8} h^{-2} L_{\odot}$ emitted at $6000 \AA$ at $z=5$. A significant fraction of galaxies were thus removed from the data by KAMM even at $z \geqslant 5$ and the detected CIB fluctuations must be explained by still fainter and more distant systems.

2) Clustering component from the remaining sources is given by the Limber equation which we write in the form (Kashlinsky 2005a):

$$
\left[\frac{q^{2} P_{2}(q)}{2 \pi}\right]^{1 / 2}=F_{\mathrm{CIB}} \Delta\left(q\left\langle d_{A}^{-1}\right\rangle\right) ; \Delta^{2}(k) \equiv \frac{1}{2 \pi} \frac{k^{2} P_{3}(k)}{c \Delta t}
$$

where $P_{3}(k)$ is the 3 -D power spectrum of the sources' clustering, $d_{A}$ is the comoving angular diameter distance and $\Delta t$ is the cosmic time interval spanned by the sources. Fig. 4 shows that the clustering strength at $\geqslant 1^{\prime}$ requires $\delta F_{\mathrm{CIB}} \sim 0.1 \mathrm{nW} / \mathrm{m}^{2} / \mathrm{sr}$. The angle of $1^{\prime}$ in the concordance cosmology subtends comoving scales of 2.2-3 Mpc at $5 \leqslant$ $z \leqslant 20$. For $\Lambda$ CDM density fields with reasonable biasing one can reach relative arcminutescale fluctuations of $\leqslant 5-10 \%$ meaning that the net CIB from sources contributing to the KAMM signal at $3.6 \mu \mathrm{m}$ is $>1-2 \mathrm{nW} / \mathrm{m}^{2} / \mathrm{sr}$.

3) Shot noise constraints. The amplitude of the shot-noise power gives a particularly strong indication of the epochs of the sources contributing to the KAMM signal. This can be seen from the expressions for the shot noise (Kashlinsky 2005a):

$$
P_{\mathrm{SN}}=\int_{m>m_{\mathrm{lim}}} f(m) d F(m) \equiv f(\bar{m}) F_{\mathrm{tot}}\left(m>m_{\mathrm{lim}}\right)
$$

Fig. [4 shows the shot noise amplitude evaluated at $3.6 \mu \mathrm{m}$ for the KAMM data as function of the source removal threshold. Because the shot-noise sets important limits on the contributions to the CIB fluctuations from remaining ordinary galaxies, we have evaluated $P_{\mathrm{SN}}$ for the data at deeper clipping limits as follows: the maps analyzed and shown in KAMM's SI were clipped to progressively lower limits until $\simeq 6 \%$ of the map remained. At deeper clipping thresholds, where one cannot compute Fourier transforms reliably, we evaluated $P_{S N}$ assuming it is proportional to the variance of the map, $\sigma_{F}^{2}$, minus that of the noise, $\sigma_{n}^{2}$, estimated by KAMM from the difference maps. (The noise, as expected, does not vary with the regions removed by deeper clipping and at $3.6 \mu \mathrm{m}$ remains $\sigma_{n} \simeq 0.5 \mathrm{nW} / \mathrm{m}^{2} / \mathrm{sr}$.) The last point may be an under-estimate as the precise value of the shot noise there may be influenced by not sufficiently well determined (for that purpose) instrument noise levels.

From Fig. [4 we conservatively adopt the fiducial value of $P_{\mathrm{SN}}=10^{-11} \mathrm{nW}^{2} / \mathrm{m}^{4} / \mathrm{sr}$ as the upper limit to the shot noise levels of the sources contributing to the measured CIB 
fluctuations. Above it was shown that the sources contributing to the fluctuations must have CIB flux greater than a few $\mathrm{nW} / \mathrm{m}^{2} / \mathrm{sr}$ and combining this with the values for $P_{\mathrm{SN}}$ shown leads via eq. 5.2 to these sources having typical magnitudes $m_{\mathrm{AB}}>29-30$ or individual fluxes below a few nJy. Such faint sources are expected to lie at $z>10$, the epoch of the first stars.

\section{Resolving the sources of the cosmic infrared background}

The CIB arises from emissions by discrete cosmological sources is therefore not really diffuse. It can and, of course, should be resolved eventually with faint/deep surveys which simultaneously have sufficiently fine angular resolution.

Salvaterra \& Ferrara (2006) have recently raised an important question of whether the existence of the Pop III era is in conflict with the recent data on $\mathrm{J}$ band dropouts (Bouwens et al 2005, Willis \& Corbin 2005). The surveys are strongly incomplete at $H>26$, but, at face value, the data indicate a paucity of $J-H>1.8$ dropouts around $H_{\mathrm{AB}} \simeq 28$ of only at most $\sim$ a few per $\operatorname{arcmin}^{2}$ (Bouwens et al 2005) and a similarly low upper limit on the dropouts at $J-N B \geqslant 0.3$ in the ZEN survey of Willis \& Corbin (2005). Is there necessarily a conflict or do these data tell us something significant about the Pop III era physics? Such theoretical computations of the counts of the Pop III sources are - for now - necessarily based on the following assumptions listed here in the decreasing order of the subjectively perceived likelihood: 1) the distribution of small scale fluctuations of the primordial density field is Gaussian; 2) the small scale power is that of the concordance $\Lambda \mathrm{CDM}$ model; 3 ) the mass function of the first objects is described well by a Press-Schechter type prescription although the effective index of the $\Lambda$ CDM power spectrum at these scales $n \rightarrow-3$ and pressure effects may be important at such low masses (cf. Springel et al 2005); 4) that the redshift, $z_{3}$, out to which Pop III era extended is $\left.z_{3}<9,5\right)$ the SED of Pop III systems is cut off at the Lyman- $\alpha$ frequency of the source epoch and the cutoff is not produced by the IGM at lower $z$ (cf. Santos et al 2002), and most crucially 6) that the efficiency of Pop III formation inside the parental halos is the same for all masses and epochs. Dropping any of the assumptions would lead to significant changes in the predicted number counts. The last assumption is particularly critical, because of the ease of both the destruction of $H_{2}$ molecules by Lyman-Werner band photons (e.g., Haiman et al. 1997) and their creation following the ionization of the surrounding nebula (Ferrara 1998; see reviews by Barkana \& Loeb 2001; Bromm \& Larson 2004).

Fig. [5 marks the filters around 1.1 and $1.6 \mu \mathrm{m}$ used by Bouwens et al (2005) and Willis \& Corbin (2005) vs an example of the emission template from Pop III stars at $z=10,12,15$. The figure shows that both surveys probe a very narrow range of redshifts: $z=12$ by Bouwens et al and $z=10$ by ZEN. Indeed, only for $z=12$ are sufficiently massive Pop III systems (the mass in Pop III stars $M_{*}>10^{6} M_{\odot}$ ) likely to produce $H \sim 27-28$ and satisfy $J-H>1.8$. (The constraints from the ZEN survey are weaker). Thus the counts problem - even with the above assumptions - provides information only for these narrow $(\Delta z<\sim 1)$ epochs. The discussion in Sec. 5.2 shows that Pop III systems have the mean flux of less than $\sim 5-10 \mathrm{nJy}$ at $3.6 \mu \mathrm{m}$; such populations cannot be detected in the above surveys. Why Pop III form in such small systems is an interesting question which is likely related to the variation of the efficiency of Pop III formation with epoch and mass.

In order to detect the faint sources responsible for the CIB fluctuations with fluxes below a few $\mathrm{nJy}$, as discussed in Sec. 5.2 embedded in the underlying sea of emissions, their individual flux must exceed the confusion limit usually taken to be $\alpha \geqslant 5$ times 
the flux dispersion produced by these emissions (Condon 1974). If these sources do not have the necessary flux levels they will be drowned in the confusion noise and will not be individually identifiable in galaxy surveys. Of course, this is precisely where CIB studies would take off. From observations one knows that confusion levels are not reached in J band until $m_{\mathrm{AB}} \sim 28$ (e.g. Gardner \& Satyapal 2002). If such sources were to contribute to the CIB required by KAMM data, at $3.6 \mu \mathrm{m}$ they had to have the average surface density of

$$
\bar{n} \sim F_{\mathrm{CIB}}^{2} / P_{\mathrm{SN}} \sim 5 \operatorname{arcsec}^{-2}\left(\frac{F_{\mathrm{CIB}}}{2 \mathrm{nWm}^{-2} \mathrm{sr}^{-1}}\right)^{2}\left(\frac{P_{\mathrm{SN}}}{10^{-11} \mathrm{nW}^{2} \mathrm{~m}^{-4} \mathrm{sr}^{-1}}\right)^{-1}
$$

In order to avoid the confusion limit and resolve these sources individually at, say, 5-sigma level $(\alpha=5)$ one would need a beam of the area

$$
\omega_{\text {beam }} \leqslant \alpha^{-2} / \bar{n} \sim 5 \times 10^{-3}\left(\frac{F_{\mathrm{CIB}}}{2 \mathrm{nWm}^{-2} \mathrm{sr}^{-1}}\right)^{-2} \operatorname{arcsec}^{2}
$$

or of circular radius below $\sim 0.04\left(F_{\mathrm{CIB}} / 2 \mathrm{nWm}^{-2} \mathrm{sr}^{-1}\right)^{-1}$ arcsec. This is clearly not in the realm of the currently operated instruments, but the JWST could be able to resolve these objects given its sensitivity and resolution.

\section{Conclusions}

Both the CIB and Population III studies are rapidly evolving into observation-rooted, if not yet high-precision, disciplines. The current situation is therefore very dynamic, but one can summarize the up-to-date constraints on the Pop III era that emerge from the recent CIB measurements as follows:

- The measurements of the mean levels of the CIB indicate that about up to a few $\%$ of baryons may have gone through Pop III. The new measurements of the $z \sim 0.1-0.2$ blazar spectra at $\sim$ a few $\mathrm{TeV}$ energies indicate that the net levels of the CIB may be not as high as the DIRBE- and IRTS-based analyses indicate. Nevertheless, these data show an evidence of absorption in excess of what can be provided by the observed galaxy populations indicating additional fluxes from fainter (and likely more distant) sources. If real, these near-IR CIB fluxes should be detectable from the future GLAST-based observations of the high $z$ gamma-ray bursts.

- The recent measurements of the CIB fluctuations allow to probe the emissions from high $z$ sources more directly. They indicate that the sources have fluxes below $\sim 10 \mathrm{nJy}$ at $3.6 \mu \mathrm{m}$, or $m_{A B}>28-29$. Such sources are likely to be located at $z>10$, the era associated with the first stars. These sources must have provided CIB levels of at least a few $\mathrm{nW} / \mathrm{m}^{2} / \mathrm{sr}$ at 3.6 to $5 \mu \mathrm{m}$ and their clustering properties have to fit the measured CIB fluctuations of $\sim 0.1 \mathrm{nW} / \mathrm{m}^{2} / \mathrm{sr}$ at arcmin scales as well as have the SED that gives flat to slowly rising CIB fluctuations with increasing wavelength.

- The above constraint on the typical flux (a few nJy) of these sources suggests that they have less than $\sim 10^{5} M_{\odot}$ in stars inside each of the haloes. This likely requires a halo-dependent efficiency if the parental haloes form out of the $\Lambda \mathrm{CDM}$ density field prior to $z \sim 10$.

- The sources contributing to the CIB fluctuations at the Spitzer-IRAC bands should be resolvable in surveys with sufficiently low noise (to measure the low fluxes) and high angular resolution (to overcome cosmological confusion). At $3.6 \mu \mathrm{m}$ this can probably be done with the future James Webb Space Telescope.

Acknowledgements. I warmly thank my collaborators on the CIB fluctuations project, 
Rick Arendt, John Mather and Harvey Moseley, for many discussions and contributions. This work was supported by NSF and NASA/Spitzer grants.

\section{REFERENCES}

Abel, T. et al 2002, Science, 295, 93

Aharonian, F. et al 2006, Nature, 440,1018

Arendt, R. \& Dwek, E. 2003, Ap.J., 585, 305

Barkana, R. \& Loeb, A. 2001, Phys. Rep., 349, 125

Bouwens, R.J., Illingworth, G., Thompson, R., \& Franx, M. 2005, Ap.J., 624, L5

Bromm, V. et al 1999, Ap.J., 527, L5

Bromm, V. \& Larson, R. 2004, ARA\&A, 42, 79

Cambresy, L. et al 2001,Ap.J.,555,563

Condon, J. 1974, Ap.J., 188, 279-286

Cooray, A. et al 2004, Ap.J., 606, 611

Dwek, E. \& Arendt, R. 1998, Ap.J.,508,L9

Dwek, E., Krennrich, F., \& Arendt, R. 2005, Ap.J., 634,155

Fazio, G. et al 2004, Ap.J.Suppl., 154, 39

Ferrara, A. 1998, Ap.J., 499, L17

Gardner, J. P. \& Satyapal, S. 2000, A. J., 119, 2589

Gorjian, V. et al 2001,536,550

Haiman, Z., Rees, M.J., \& Loeb, A. 1997, Ap.J., 476, 458

Heger, A. et al 2003, Ap.J.,591, 288

Kashlinsky, A. 2005a, Phys. Rep., 409, 361-438

Kashlinsky, A. 2005b, Ap.J., 633, L5

Kashlinsky, A., Arendt, R., Mather, J.C. \& Moseley, S.H. 2005, Nature, 438, 45 (KAMM)

Kashlinsky, A., Arendt, R., Gardner, J.P., Mather, J.C., \& Moseley, S.H. 2004, Ap.J., 608, 1

Kashlinsky, A., Mather, J.C., Odenwald, S. \& Hauser, M. 1996a, Ap.J.,470,681

Kashlinsky, A., Mather, J.C., \& Odenwald, S. 1996b, Ap.J.,479, L9

Kashlinsky, A. \& Odenwald, S. 2000, Ap.J.,528,74

Madau, P. \& Pozzetti, L. 2000,MNRAS,312,L9

Madau, P. \& Silk, J. 2005,MNRAS, 359, L37

Magliochetti, M., Salvaterra, R., Ferrara, A. 2003, MNRAS,342,L25

Matsumoto, M. et al 2005, Ap.J., 626, 31

Rees, M.J. 1978, Nature,275,35

Salvaterra, R. \& Ferrara, A. 2003, MNRAS,339,973

Salvaterra, R. \& Ferrara, A. 2006, MNRAS,367, L11

Santos, M.R., Bromm, V., Kamionkowski, M. 2002,MNRAS,336,1082

Savage, R.S. \& Oliver, S. 2005, astro-ph/0511359

Schaerer, D. 2002, Aston. Astrophys., 382,28

Siess, L., Livio, M. \& Lattanzio, J. 2002, Ap.J., 570, 329

Spergel, D. et al 2006, Ap.J., submitted. astro-ph/0603449

Willis, J.P. \& Courbin, F. 2005, MNRAS, 357, 1348

Wright, E.L. \& Reese, E.D. 2000, Ap.J., 545, 43 